\title{
A Randomized, Double Blind Study to Evaluate the Efficacy of Palono- setron with Dexamethasone Versus Palonosetron Alone for Prevention of Post-Operative Nausea and Vomiting in Subjects Undergoing Bariatric Surgeries with High Emetogenic Risk
}

\author{
S. Didehvar*, J.D. Viola-Blitz, M. Haile, L. Franco, R. Kline, M. Kurian, G. Fielding, C. Ren and \\ A. Bekker
}

Department of Anesthesiology, Department of Surgery, NYU Langone Medical Center, New York, NY. A. Chu; NYU Department of Psychology, New York, NY

\begin{abstract}
Introduction: Postoperative (PONV) and postdischarge (PDNV) nausea and vomiting are common (60-70\%) after bariatric surgery. Palonosetron (Pal), a novel 5-HT3 antagonist, is an effective antiemetic with a prolonged duration of action in the setting of PDNV. We hypothesized that combination therapy with Palonosetron (Pal) and dexamethasone (Dex) would improve treatment in comparison to Palonosetron alone in patients at high risk for PONV.

Methods: In this study, patients undergoing bariatric laparoscopic surgery under general anesthesia, a subgroup of a larger Phase IV clinical trial of patients who had laparoscopic surgery, were randomized to $8 \mathrm{mg}$ Dex $+0.075 \mathrm{mg}$ Pal or saline + 0.075mg Pal. Data was collected postoperatively at 2, 6, 24 and 72 hrs. A Functional Living Index-Emesis (QOL-FLIE) test was administered at 96 hrs.

Results: We enrolled 76 ASA 1-2 patients with at least 3 PONV risk factors. Both randomization groups had a low incidence of vomiting in the PACU (Pal, 0.0\%; Pal + Dex, 5.4\%) as well as at 72 hours ( $0.0 \%$ both groups). Complete response (no vomiting, no rescue medication) was not different between treatment groups at any time intervals. Cumulative success rates over the entire 72 hrs were 60.4\% (Pal alone) vs. 60.0\% (Pal + Dex). Nausea scores (4 point ordinal scale) were not different between groups for any time intervals. Cumulative success scores for nausea (score = "none"; 0-72 hrs) were $41.9 \%$ for the Pal group, and 55.2\% for the Pal+ Dex group. The Pal + Dex group showed a trend toward greater satisfaction on the QOL-FLIE scores with the greatest differences in the "nausea domain".

Discussion: The combination therapy (Pal + Dex) did not significantly reduce the incidence of PONV or PDNV when compared with Pal alone although a trend was observed indicating the possible increased efficacy of multi-drug therapy. There was no change in comparative efficacy over 72 hrs, possibly due to the low incidence of PDNV in both groups.
\end{abstract}

Keywords: Post-discharge nausea, post-operative nausea and vomiting, bariatric surgery.

\section{INTRODUCTION}

Postoperative (PONV) and postdischarge (PDNV) nausea and vomiting are common occurrences (60-70\%) after bariatric surgery [2]. Both complications may lead to significant morbidity after laparoscopic banding surgery due to suture disruption, an increased risk of aspiration of gastric contents, and electrolyte imbalances3. Anticipating or treating PONV alone is not sufficient because approximately $36 \%$ of patients who experience PDNV do not experience PONV [3, 4]. Palonosetron (Pal), a novel 5-HT3 antagonist, is an effective antiemetic in the setting of PONV that also has advantages in treating PDNV due to its prolonged duration

*Address correspondence to this author at the Department of Anesthesiology, NYU Langone Medical Center, $5501^{\text {st }}$ Ave RR 605, New York, NY 10016; Tel: 212-263-0667; Fax: 212-263-3211;

E-mail: Sorosch.Didehvar@nyumc.org of action. Palonosetron exhibits greater binding affinity and has a longer half-life than older 5-HT3 antagonists, possibly due to its binding of 5HT-3 receptors in an allosteric, positively cooperative manner [5-7]. Prior studies have demonstrated the advantages of a multimodal approach to the treatment of PONV, including a reduction in the incidence of PONV in high risk patients with the combination of 5-HT3 receptor antagonists and dexamethasone [8, 9]. However, dexamethasone may increase the rate of surgical complications including infection and irritation of the gastric mucosa. We hypothesized that the addition of dexamethasone is not warranted in patients treated with palonosetron, a highly effective long acting drug for prevention of PONV. Our recent study demonstrated that the combination therapy of palonosetron and dexamethasone did not improve the incidence of PONV or PDNV when compared with palonosetron alone in subjects with high emetogenic risk undergoing laparo- 
scopic surgery [1]. Dexamethasone could be especially harmful in patients undergoing bariatric surgery due to the high incidence of gastric reflux in that patient population. Therefore we analyzed the data from our larger phase IV trial to focus on patients undergoing bariatric surgery. We hypothesized that the addition of dexamethasone is not required for relief of PONV and PDNV when patients are treated with palonosetron.

\section{METHODS}

After IRB approval and written informed consent, patients with three or more risk factors for PONV scheduled to undergo elective laparoscopic gastric banding surgery under general anesthesia were enrolled in this single-center, prospective, double blind study. Those risk factors included female gender, history of motion sickness (MS) or PONV, nonsmoking history, and the use of postoperative opioids. All patients received $0.075 \mathrm{mg}$ IV palonosetron. Thirty seven patients were randomized to receive $8 \mathrm{mg}$ IV dexamethasone (Pal + Dex) upon induction of general anesthesia. Thirty nine patients were randomized to receive an equivalent volume of saline (Pal). Patients who were pregnant, had received antiemetics within 24 hours of surgery, experienced retching or vomiting prior to surgery, were on chronic steroid therapy, were immunocompromised, or had a documented allergy to either 5-HT3 receptor antagonists or dexamethasone were excluded from the study. Data was collected at defined postoperative times (2, 6, 24 and $72 \mathrm{hrs).} \mathrm{All} \mathrm{patients} \mathrm{also} \mathrm{com-}$ pleted an 18 question-QOL-FLIE (Functional Living IndexEmesis) instrument at 96 hrs [10].

General anesthesia was induced with $2-4 \mathrm{mg} / \mathrm{kg}$ of propofol and tracheal intubation was facilitated with either 0.6 $\mathrm{mg} / \mathrm{kg}$ of rocuronium or $1 \mathrm{mg} / \mathrm{kg}$ of vecuronium. The study drug was administered immediately after induction. Anesthesia was maintained with Sevoflurane in a combination of oxygen and air. Fentanyl (2-10 mcg/kg) with or without ketorolac $30 \mathrm{mg}$ IV/30 mg IM was administered for analgesia. Muscle paralysis was reversed at the end of the surgery with $0.04-0.07 \mathrm{mg} / \mathrm{kg}$ of neostigmine and $0.4-1 \mathrm{mg}$ of glycopyrrolate. Intraoperative monitoring included heart rate via a 3 lead electrocardiogram, non-invasive blood pressure cuff, oxygen saturation by pulse oximetry, and $\mathrm{PETCO}_{2}$. Nausea and vomiting data were collected at 2, 6, 24 and 72 hrs. Nausea was determined using a self reported 4 point ordinal scale: none, mild, moderate and severe. Treatment success was defined as all "zero" scores at every time period. Suc- cess for emesis was scored when (no vomiting) were recorded at all hours measured for the scoring interval, and no rescue medication was given for that interval. Any reports of nausea that occurred between $0-6$ hours post-operatively was considered postoperative nausea (PON), while reports of nausea between the times of 6-72 hours post surgery were defined as postdischarge nausea (PDN). Vomiting up to 6 hours post surgery was defined as early vomiting, whereas any episodes of emesis in the interval of 6-72 hours post surgery was described as delayed vomiting. The time course of 0-6 hours was chosen to reflect the period during which the patient was less mobile (including time spent in PACU), versus 6-72 hours when patients were increasingly mobile (i.e. after discharge to the hospital floor).

No other anti-emetic medications were used during the operation. Rescue anti-emetic use was permitted. Rescue anti-emetics used included metoclopramide. Rescue antiemetics were given during the PACU stay or upon discharge from the PACU upon the patient's request or after an episode of vomiting. However, 5-HT3 receptor antagonist use was not permitted. A complete response (CR) was defined as no vomiting and no rescue medication for any time interval. All patients completed an 18 question QOL-FLIE (Functional Living Index-Emesis) instrument at 96 hrs [13]. For statistical testing of the null hypothesis that the nausea responses of the two treatment groups were equal, we used an ordinal regression model (which included age, BMI; SPSS v 18).Data was also grouped as a binary (success/failure) and analyzed with chi square or Fisher's Exact test. $\mathrm{P}<0.05$ was accepted as statistically significant. The study was powered $(n=76)$ to be able to detect a drop in complete response from $90 \%$ to $70 \%$ while comparing Pal+Dex to Dex alone (2-sided alpha $=0.05$; power $=80 \%$ ). Percocet was the anesthetic used postoperatively and there were no significant differences in its use or dosage. For example, $72 \%$ of the Pal + Dex group received the drug while $67 \%$ of the Pal group received the drug.

\section{RESULTS}

We enrolled 76 patients, ASA 1-2, with at least 3 PONV risk factors, who were undergoing laparoscopic bariatric surgery as outpatients (23 hour hospital admission). Table $\mathbf{1}$ demonstrates that there was no difference in patient demographics between groups, including BMI (Pal: 44.1_6.8; Pal + Dex: 41.9+6.3; $\mathrm{p}=0.162$ ).

Both groups had a low incidence of vomiting at $0-2 \mathrm{~h}$ (Pal: 0.0\%; Pal + Dex: 5.4\%) at 2-6 h (Pal: 9.1\%; Pal + Dex:

Table 1.

\begin{tabular}{|c|c|c|c|c|}
\hline Parameter & Treatment Pal alone & Treatment Pal + Dex & Total & 61 \\
\hline \hline Female & 31 & 30 & 15 & $35.4 \pm 8.7$ \\
\hline Male & 8 & 7 & $43.1+6.6$ & .822 \\
\hline Age & $35.2 \pm 9.2$ & $41.9+6.3$ & 36 & .162 \\
\hline BMI & $44.1+6.8$ & 37 & \\
\hline
\end{tabular}

76 patients were randomized with 39 in the group that received Pal alone and 37 in the group that received Pal + Dex. The population includes 61 females and

15 males. We compared the age (mean \pm SD) and BMI of the two treatment groups, and found no significant differences. 
6.5\%) and at 6-72 h (Pal: 6.1\%; Pal + Dex: 3.4\%). There were no differences in incidence of vomiting by treatment group ( $\mathrm{p}>0.32$ ) for any of the designated time intervals $(0-2$ hrs, 2-6 hrs, 6-72 hrs).

Fig. (1) shows the proportion of complete responses (no vomiting and no rescue medication required) by treatment group at different time intervals. There were no differences by treatment group ( $\left.>0.39 ; \chi^{2}<0.72\right)$ for any of the designated time intervals (0-2 h: Pal $=87 \%$ vs. Pal + Dex $=86 \%$, 2-6 h: Pal $=90 \%$ vs. Pal + Dex $=84 \%, 6-72$ h: $\mathrm{Pal}=84 \%$ vs. $\mathrm{Pal}+$ Dex $=83 \%, 0-72 \mathrm{~h}: \mathrm{Pal}=68 \%$ vs. $\mathrm{Pal}+\mathrm{Dex}=62 \%)$.

Cumulative success scores for nausea (score = "none"; 072 hrs) were $41.9 \%$ for Pal group, and 55.2\% for Palonosetron + Dexamethasone group. Fig. (2) displays the rates of mild, moderate, or severe nausea between groups at varying time intervals. The overall incidence of nausea was low and not statistically different between groups, both in the immediate postoperative period $(0-2 \mathrm{~h})(\mathrm{Pal}=1.6 \%$, Pal + Dex $6.7 \%)$ and in the interval 6-72 hours post-procedure $(\mathrm{Pal}=$ 4.2\% vs. Pal + Dex $6.5 \%$ ).

Analysis of the QOL questionnaire indicates that the $\mathrm{Pa}$ lonosetron + Dexamethasone group showed a trend toward greater satisfaction on the QOL-FLIE scores with the greatest differences in the "nausea domain"; however this did not reach statistical significance (Fig. 3). For each of 18 quality of life questions, we compared mean scores by treatment group. None of the individual question scores were significantly different by treatment group with the exception of question 3 ("Has nausea affected your ability to make a meal or do minor household repairs during the past 3 days?”, $\mathrm{p}=$ 0.039; 2 tailed t-test).

Quality outcomes are equal or better for the Palonosetron + Dexmethasone group. However, summing all the scores for each patient, we found no difference by treatment group (t-test; $\mathrm{p}=0.121$ ). Multivariate statistics also failed to show treatment related differences.

\section{DISCUSSION}

The incidence of PONV in the PACU was low compared to historical controls in this study of PONV and PDNV in patients undergoing bariatric surgery[4], however combination therapy with dexamethasone did not significantly reduce the incidence of PONV or PDNV compared with palenosetron alone. There was no change in comparative efficacy over 72 hrs, possibly due to the low incidence of PDNV in both groups. This is in agreement with our previous larger Phase IV trial upon which this bariatric subgroup study is based. Our findings in both the bariatric subgroup and the larger group are in contrast to the findings of Mendes et al. [12] who found a significant decrease in the incidence of

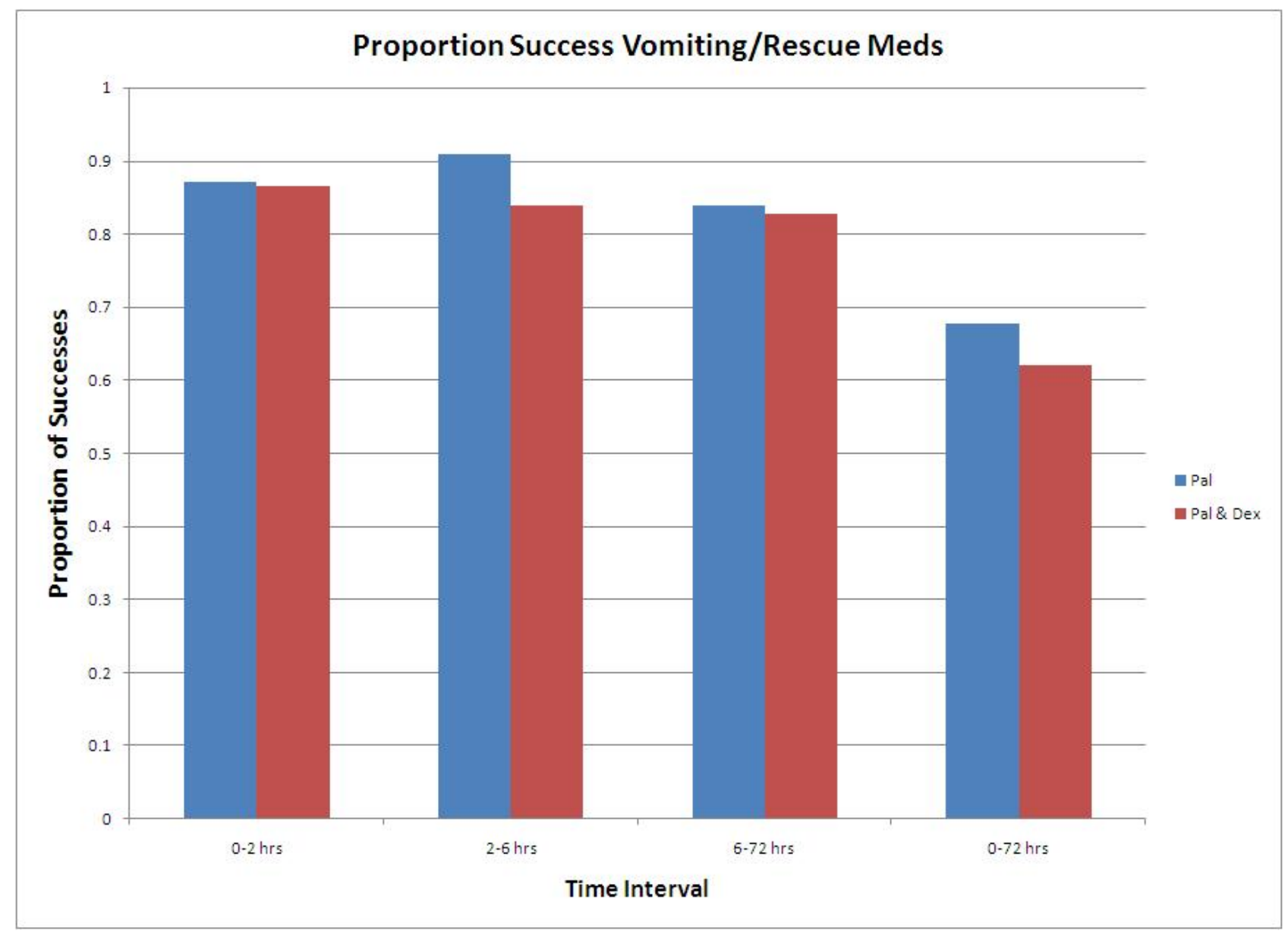

Fig. (1). The proportion of successes (no vomiting and no rescue medication given) for the two treatment groups (Palonosetron - blue bars; Palonosetron plus Dexamethasone - red bars) are plotted for 4 time intervals (shown on horizontal axis). 


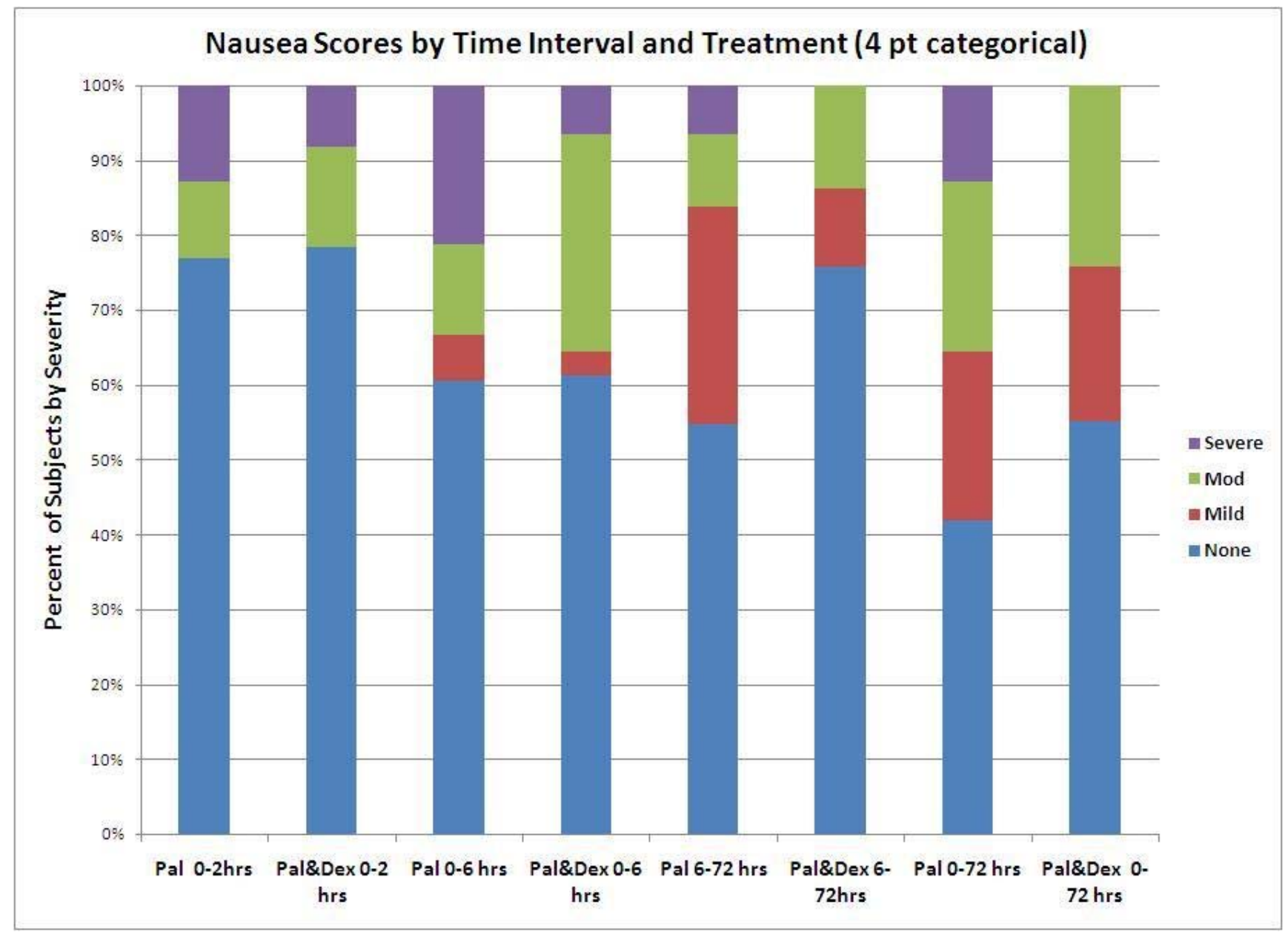

Fig. (2). Subject nausea reports by category: none, score $=0$; mild, $0<$ score $<=1$; moderate, $1<$ score $<=3$; and severe, $>3$. The bars are identified on the horizontal axis by time interval and treatment group.

PONV in the first $24 \mathrm{~h}$ post laparoscopic bariatric surgery in patients who had received the combination of ondansetron + dexamethasone compared to those patients who had received ondansetron alone. Furthermore, this study found that there was no change in comparative efficacy in the interval 0-72 hours post-surgery. This was most likely due to the low incidence of PDNV in both groups. The proportion of treatment successes (no vomiting and no rescue medication) was compared for the two treatment groups, and indicated no therapeutic benefit in adding dexamethasone. The incidence of vomiting was very low in both groups. Using binary analysis (success/failure), there was no trend in the nausea scores to suggest that dexamethasone improved outcome when added to palonosetron. However, using ordinal regression analysis, and examining the later time period (6-72 hrs), results were suggestive of BMI and treatment group being predictors of nausea in this bariatric population. This may be due to the fact the palonosetron dose is a minimally effective dose for normal weight subjects [11]. Therefore the drug could lose effectiveness over time for the heaviest of the bariatric subjects. If confirmed, such a finding would indicate need for reconsideration of the bariatric dose for surgical prophylaxis.

The negative impact of PONV on patient recovery is well recognized: not only does it cause patient discomfort and delay discharge from the PACU in patients who undergo ambulatory surgery, it can lead to electrolyte disturbances, aspiration, or even suture disruption. Although less is currently known about the risk factors for PDNV and the optimal strategy for its treatment, the significance of PDNV's impact on patients who undergo outpatient surgery cannot be underestimated. PDNV appears to be more common than previously thought, and has become more important as an increasing number of surgical procedures are performed on an outpatient basis. Palonosetron has been documented to be effective in the prevention and treatment of both PONV and PDNV as well as the prevention and treatment of chemotherapy induced nausea and vomiting. Although the combination of dexamethasone with other shorter acting 5-HT3 antagonists has been documented to be effective for the treatment of PONV [2] the combination of palonosetron with dexamethasone had not been studied to date. The results of our study suggest that palonosetron may be unique in that the administration of a single dose of this drug was effective for preventing both PONV and PDNV. In addition, the administration of other anti-emetic agents did not further decrease the incidence of PONV and PDNV.

This finding has important consequences. In any multidrug regimen, there is an increased risk of adverse reactions: either to a drug or to a combination of drugs. In addition the 


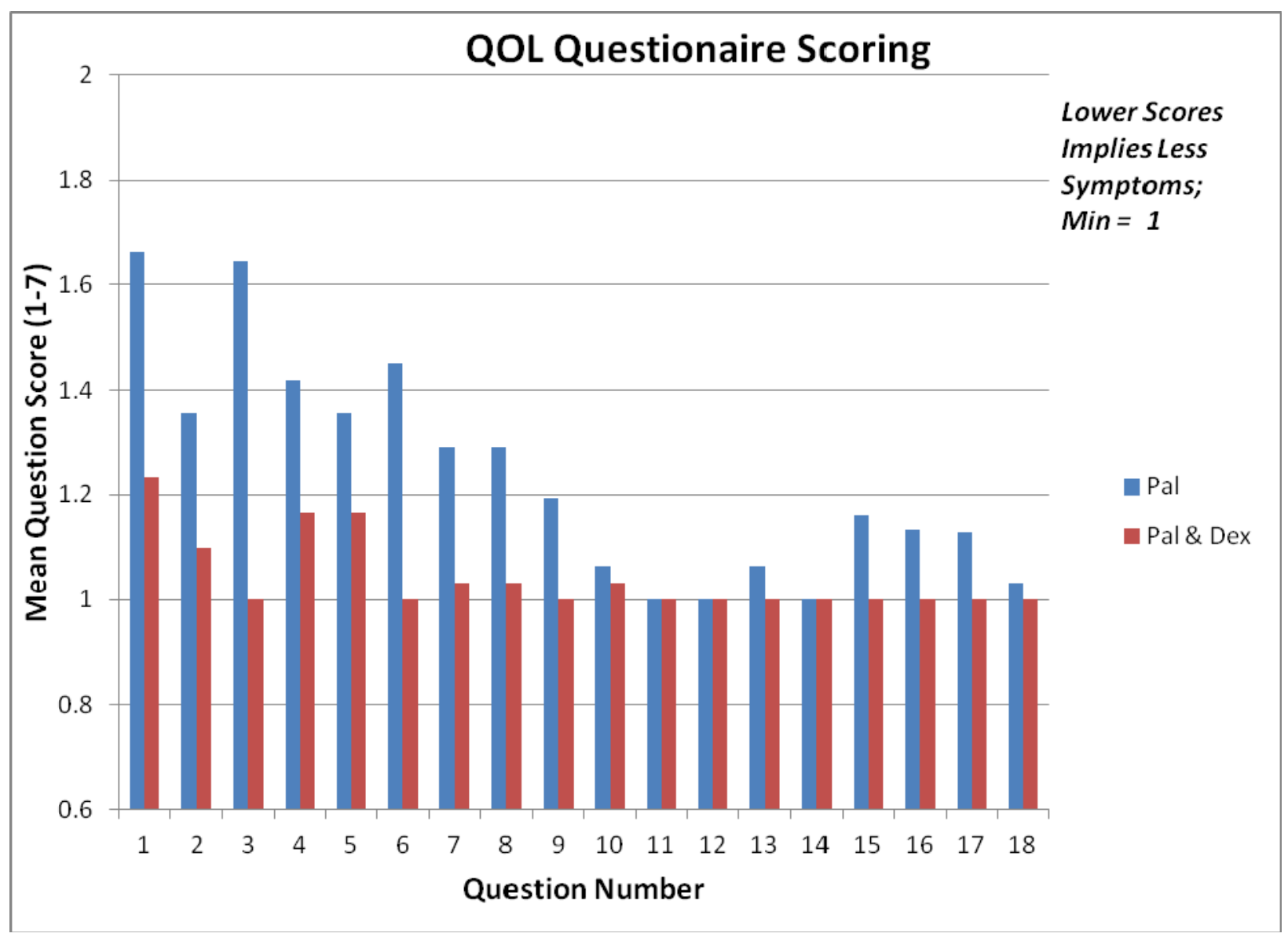

Fig. (3). Individual 2 tailed t-tests showed no significant difference in the scores for any particular questions with regard to randomization with the exception of Question 3 ( $\mathrm{p}=0.039$, before multiple comparison correction). Examining total scores over each patient, and then comparing the means by treatment group using t-tests, we found no significant difference.

patient is exposed to potential side effects from each drug that is administered. Palonosetron, at any dose, has not been found to prolong the QTc interval in contrast to the older 5HT3 receptors [13]. If administering one drug is as effective as a multi-drug regimen, patient safety is increased without sacrificing efficacy. Furthermore, palonosetron's long duration of action is beneficial because one dose can be administered to the patient at the time of surgery requiring no further doses upon discharge from the hospital or afterwards. Palonosetron therapy may lead to a decrease in the incidence of PDNV as well as the costs associated with the prevention and treatment of PDNV.

Our results are consistent with the finding of other investigators who have studied the non-bariatric patient population. Kovac et al. [11] reported a complete response (CR) rate (no nausea and no vomting at any time interval) of 56\% of patients who had received a dose of palonosetron $0.075 \mathrm{mg}$. Candiotti [13] reports a CR rate of $43 \%$ at $0-24 \mathrm{~h}$ after surgery and $49 \%$ at $24-72 \mathrm{~h}$ after surgery. This is consistent with the $60 \%$ success rate in the Pal group that we report in our study. In addition, Kovac et al report that $27 \%$ of patients who had received $0.075 \mathrm{mg}$ of pal required rescue therapy, whereas we found that of the patients who received palonosetron $0.075 \mathrm{mg}$, only $16 \%$ required rescue therapy. Although Kovac et. al. suggested that palonosetron's efficacy appears to be mainly in the first 24 hours, our study reported a low incidence of nausea $45 \%$ (Pal group) and 23\% (Pal + Dex group) 6-72 h postoperatively, indicating that pal's efficacy may in fact extend beyond the first $24 \mathrm{~h}$ time period. This has important consequences for ambulatory patients who would otherwise experience PDNV after discharge.

Henzi et al. [14] reported that dexamethasone reduced the incidence of PDNV; however their study defines "late" PONV or PDNV as occurring $24 \mathrm{~h}$ post-operatively. In contrast, our study examines the effect of dexamethasone beyond the $24 \mathrm{~h}$ post surgery period. It is important to note that at the $72 \mathrm{~h}$ time interval, although there was a trend toward a higher percentage of success in the Pal + Dex group, this was not significantly different than the percentage of successes in the group that received $\mathrm{Pal}$ alone.

Our trial has several limitations. The modest sample size may not be sufficient for a robust evaluation of the treatment effect on all end-points and at all time intervals for the purposes of statistical analyses. For example, the low rate of vomiting ( $0 \%$ in both groups) noted in this trial during the 24-72 hour interval after surgery precluded us from meaningful comparison of the groups. Although this study was placebo controlled with regard to the administration of dexamethasone, the lack of an active comparison between palonosetron, palonosetron $+\operatorname{dex}$ and a third arm where no anti-emetic was administered limits this study's ability to be 
The questions above with the largest difference were: 1] quantity of nausea; 3] ability to make a meal or do minor household repairs; 6] willingness to spend time with family/ friends; 7] affected daily functioning; and 9] imposed hardship on others. All were in the nausea domain. (Complete list of questions follow - chart 1.)

\section{Chart 1: Quality of Life Questionnaire}

1. How much nausea have you had in the past 3 days?

2. Has nausea affected your ability to maintain usual recreation or leisure activities in the past 3 days?

3. Has nausea affected your ability to make a meal or do minor household repairs during the past 3 days?

4. How much has nausea affected your ability to enjoy a meal in the past 3 days?

5. How much has nausea affected your ability to enjoy liquid refreshment in the past 3 days?

6. How much has nausea affected your ability to enjoy liquid refreshment in the past 3 days?

7. Has nausea affected your daily functioning in the past 3 days?

8. Rate the degree to which your nausea has imposed a hardship on you (personally) in the past 3 days?

9. Rate the degree to which your nausea has imposed a hardship on those closest to you in the past 3 days?

10. How much vomiting have you had in the past 3 days?

11. Has vomiting affected your ability to maintain usual recreation or leisure activities in the past 3 days?

12. Has vomiting affected your ability to make a meal or do minor household repairs during the past 3 days?

13. How much has vomiting affected your ability to enjoy a meal in the past 3 days?

14. How much has vomiting affected your ability to enjoy liquid refreshment in the past 3 days?

15. How much has vomiting affected your willingness to see and spend time with family and friends, in the past 3 days?

16. Has vomiting affected your daily functioning in the past 3 days?

17. Rate the degree to which your vomiting has imposed a hardship on you (personally) in the past 3 days.

18. Rate the degree to which your vomiting has imposed a hardship on those closest to you in the past 3 days.

compared directly with published placebo-controlled trials of older 5-HT3 receptor antagonists. In addition, end-points were measured during preset time intervals $(0-6 \mathrm{~h}, 6-72 \mathrm{~h})$, rather than evaluated by occurrence in a particular setting such as the PACU (0-2h) or post-PACU (2-24 h) time intervals. Furthermore, because the risk factors for developing PDNV are not well established and may not be the same as those for PONV, it is difficult to make predictions about how to best prevent PDNV.

The incidence of PONV in the PACU was low compared to other studies. For example, Breitfield et al. [15] observed post-operative opioid-induced emesis in about one-third of patients while the occurrence of vomiting was one-half of that number. In our study, although approximately $2 / 3$ patients in either group (no significance between groups in terms of dosage and treatment), required post-operative opioid therapy, the incidence of PONV remained relatively low in possibly because of treatment for that condition, the length of surgery, different types of surgery and intra-operative anesthetics used.

Palonosetron's long duration of action may be of particular benefit because one dose can be administered to the patient at the time of surgery, and no further doses are required upon discharge from the hospital or afterwards. Our data are in accordance with the view that a combination of a 5-HT3 antagonist and dexamethasone decreases nausea in the laporoscopic bariatric surgery population which may translate to greater patient satisfaction. However, additional research is required to evaluate whether or not this finding is clinically relevant.

\section{CONFLICT OF INTERSEST}

The authors confirm that this article content has no conflicts of interest.

\section{ACKNOWLEDGEMENTS}

Funding for this investigator-initiated clinical trial was provided by the sponsor Eisai Inc.

\section{SOURCE OF SUPPORT}

Eisai Inc.

\section{REFERENCES}

[1] Blitz JD, Haile M, Kline R, et al. A randomized double blind study to evaluate efficacy of palonosetron with dexamethasone versus palonosetron alone for prevention of postoperative and postdischarge nausea and vomiting in subjects undergoing laparoscopic surgeries with high emetogenic risk. Am J Ther 2012; 19(5): 324-9.

[2] Moussa AA, Oregan PJ. Prevention of postoperative nausea and vomiting in patients undergoing laparoscopic bariatric surgerygranisetron alone vs granisetron combined with dexamethasone/ droperidol. Middle Eastern J Anesthesiol 2007; 19: 357-67.

[3] Carroll NV, Miederhoff P, Cox FM et al. Postoperative nausea and vomiting after discharge from outpatient surgery centers. Anesth Analg 1995; 80: 903-9. 
[4] Le TP, Gan TJ. Update on the management of postoperative nausea and vomiting and postdischarge nausea and vomiting in ambulatory surgery. Anesthesiol Clin 2010; 28: 225-49.

[5] Rojas C, Stathis M, Thomas AG, et al. Palonosetron exhibits unique molecular interactions with the 5-HT3 receptor. Anesth Analg 2008; 107: 469-78.

[6] Tang J, D’Angelo R, White PF, et al. The efficacy of RS-25259, a long-acting selective 5-HT3 receptor antagonist, for preventing postoperative nausea and vomiting after hysterectomy procedures. Anesth Analg 1998; 87: 462-7.

[7] Gan TJ. Selective serotonin 5-HT3 receptor antagonists for postoperative nausea and vomiting. Are they all the same? CNS Drugs 2005; 19: 225-38.

[8] Apfel CC, Korttila K, Abdalia M. A factorial trial of six interventions for the prevention of postoperative nausea and vomiting. $\mathrm{N}$ Engl J Med 2004; 350: 2441-51.

[9] Scuderi PE, James RL, Harris L, Mims GR. Multimodal antiemetic management prevents early postoperative vomiting after outpatient laparoscopy. Anesth Analg 2009; 91: 1408-14.

[10] Decker M, DeMeyer ES, Kisko D. Measuring the maintenance of daily life activities using the functional living index-emesis (FLIE) in patients receiving moderately emetogenic chemotherapy. J Support Oncol 2006; 4: 35-41.

[11] Kovac AL, Eberhart L, Kotarski J, Clerici G, Apfel C. A randomized, double-blind study to evaluate the efficacy and safety of three different doses of palonsetron versus placebo in preventing postoperative nausea and vomiting over a 72-hours period. Anesth Analg 2008; 107: 439-44.

[12] Nespeca MM, de Souza MR, Nogueira da CMF. Prophylaxis of postoperative nausea and vomiting in morbidly obese patients undergoing laparoscopic gastroplasties. A comparative study among three methods. Revista Brasileira de Anesthesiologia 2009; 59: 570-6.

[13] Candiotti KA, Kovac AL, Melson TI, et al. A randomized, doubleblind study to evaluate the efficacy and safety of three different doses of palonsetron versus placebo in preventing postoperative nausea and vomiting. Anesth Analg 2008; 107: 445-51.

[14] Henzi I, Walder B, Tramer MR. Dexamethasone for the prevention of postoperative nausea and vomiting: A quantitative systematic review. Anesth Analg 2000; 90: 186-94.

[15] Breitfeld C, Peters J, Vockel T, Lorenz C, Eikermann M. Emetic effects of morphine and piritramide. Br J Anaesth 2003; 91: 21823.

Received: October 19, 2012

Revised: November 26, 2012

Accepted: December 07, 2012

(c) Didehvar et al.; Licensee Bentham Open.

This is an open access article licensed under the terms of the Creative Commons Attribution Non-Commercial License (http://creativecommons.org/licenses/ by-nc/3.0/) which permits unrestricted, non-commercial use, distribution and reproduction in any medium, provided the work is properly cited. 